\title{
Statistical properties of states in QED with unstable vacuum
}

\author{
S.P. Gavrilov*, D.M. Gitman’ and A.A. Shishmarev ${ }^{\ddagger}$
}

September 13, 2021

\begin{abstract}
We study statistical properties of states of massive quantized charged Dirac and Klein-Gordon fields interacting with a background that violates the vacuum stability, first in general terms and then for a special electromagnetic background. As a starting point, we use a nonperturbative expression for the density operators of such fields derived by Gavrilov et al [S.P. Gavrilov, D.M. Gitman, and J.L. Tomazelli, Nucl. Phys. B 795, 645 (2008)]. We construct the reduced density operators for electron and positron subsystems and discuss a decoherence that may occur in the course of the evolution due to an intermediate measurement. By calculating the entropy we study the loss of the information in QED states due to partial reductions and a possible decoherence. Next, we consider the so-called $T$-constant external electric field as an external background. This exactly solvable example allows us to calculate explicitly all statistical properties of various quantum states of the massive charged fields under consideration.
\end{abstract}

PACS numbers: 12.20.Ds, 03.65.Ud

Keywords: Density operator; entropy; entanglement; particle creation

\section{Introduction}

It is known that pure states of a quantum system provide us with the maximum possible information about this system in contrast with mixed states of the same quantum system. A measure of the information loss of a quantum state can be identified by the entropy of such a state. Any unitary evolution does not change the entropy of a quantum state, and possible violations of the unitary evolution can be registered as a change of the entropy. On the other hand, the entanglement is an essentially quantum property associated with the quantum non-separability of parts of a composite system. It also can be evaluated as a specific quantum entropy. Entangled states became a powerful tool in studying principal questions in quantum theory and in quantum computation and information theories [1, 2, 3, 4,. Despite a number of publications devoted to the entropy and entanglement of quantum states, for the related characteristics to be fully understood, more examples of various special systems need to be considered not only in nonrelativistic quantum mechanics, but in quantum field theory (QFT), as well. This explains recent interest in studying quantum entanglement and entropy of QFT systems with an unstable vacuum, i.e., with strong external backgrounds that may create particles from the vacuum (see, e.g., [5, 6, 7, 8]). In this article we would

*Department of Physics, Tomsk State University, Lenin ave. 36, 634050 Tomsk; Department of General and Experimental Physics, Herzen State Pedagogical University of Russia, Moyka embankment 48, 191186 St. Petersburg, Russia; e-mail: gavrilovsergeyp@yahoo.com

${ }^{\dagger}$ Department of Physics, Tomsk State University, Lenin ave. 36, 634050 Tomsk; P.N. Lebedev Physical Institute, Russian Academy of Sciences, 119991 Moscow, Russia; and Institute of Physics, University of São Paulo, CP 66318, CEP 05315-970 São Paulo, São Paulo, Brazil; gitman@if.usp.br

${ }^{\ddagger}$ Institute of Physics, University of São Paulo, CP 66318, CEP 05315-970 São Paulo, São Paulo, Brazil; ; e-mail: a.a.shishmarev@mail.ru 
like to attract attention to the fact that by studying QFT systems with an unstable vacuum we create the possibility to approach problems characterized by the loss of information, quantum entanglement, and the entropy change in a very close relation. We present two points that explain this assertion. Let a quantized charged Dirac or Klein-Gordon (KG) field 1 interact with a strong uniform external electric field. Such a system is a QFT with an unstable vacuum, which means that the electric field creates electron-positron pairs from the vacuum. Particle creation from the vacuum by strong electromagnetic, Yang-Mills, and gravitational fields is a well-known nonlinear quantum phenomenon that has many applications in modern high energy physics. Its theoretical study has a long story that is described in numerous works, see for example Refs. 11, 9, 12, 13, 10, 14, 15. The creation of charged particles from the vacuum by strong electriclike fields needs superstrong field magnitudes compared with the Schwinger critical field $E_{\mathrm{c}}=m^{2} c^{3} / e \hbar \simeq 1.3 \times 10^{16} \mathrm{~V} \cdot \mathrm{cm}^{-1}$ [16. Nevertheless, recent progress in laser physics allows one to hope that this effect will be experimentally observed in the near future even in laboratory conditions (see Ref. [17] for a review). Electron-hole pair creation from the vacuum (which is an analog of the electronpositron pair creation from the vacuum) was recently observed in graphene by its indirect influence on the graphene conductivity [18] (the conductivity of graphene modified by the particle creation was calculated in [19]; some other relevant effects may be found in [20]). The particle creation effect in a strong uniform external electric field has an additional important feature. The external field not only creates the pairs from the vacuum, but produces two subsystems, well separated in space: the created electrons and the created positrons. States of each subsystem are described by the corresponding density matrices. Such density matrices originally were derived in Refs. [21, 15]. Here it is interesting to study the quantum entanglement of both subsystems and its measure by calculating the corresponding entropy. A change of the entropy of QFT systems with an unstable vacuum and a quantum entanglement of the above mentioned subsystems can occur also due to some decoherence processes. In the case under our consideration these might be intermediate measurements or collisions with some semiclassical objects (e.g., with well-known impurities in the graphene).

In the present article, we study the above-mentioned characteristics, that is, statistical properties of states of massive quantized charged Dirac or K-G fields with a background that violates the vacuum stability, first in general terms and then considering a specific external electromagnetic background. As a starting point, we use a general nonperturbative expression for the density operators of such fields derived in Ref. [15. In Sec. 2 we discuss such operators with different initial conditions. Reduced density operators for electron and positron subsystems are derived in Sec. 3 . In Sec. 4 we study a decoherence that may occur in the course of the evolution due to an intermediate measurement and the corresponding modifications of the complete and the reduced density operators. In Sec. 5 , when calculating the entropy, we study the loss of information in the QED states due to partial reductions and possible decoherence. In Sec. [6 we consider quantized Dirac or KG fields with the socalled $T$-constant external electric field. This exactly solvable example allows us to calculate explicitly all statistical properties of different quantum states of the latter massive fields. In the Appendix we briefly recall a nonperturbative formulation of QED with strong timedependent electric-like background that is used in our calculations.

\section{General density operator}

It is convenient to introduce a generating operator $\check{R}(J)$ that allows one to construct density operators $\check{\rho}$ with different initial conditions (different initial states at the initial time instant

\footnotetext{
${ }^{1}$ As basic particles in both cases we consider electrons with the charge $q=-e, e>0$, whereas their antiparticles are positrons. In the KG case both electrons and positrons are spinless.
} 
$\left.t_{\text {in }}\right)$. This generating operator has the following form:

$$
\begin{aligned}
& \check{R}(J)=Z^{-1}(J) \underline{\check{R}}(J), \operatorname{tr} \check{R}(J)=1, Z(J)=\operatorname{tr} \underline{\check{R}}(J), \\
& \check{\underline{R}}(J)=: \exp \left[\sum_{n}\left[a_{n}^{\dagger} \text { (in) }\left(J_{n,+}-1\right) a_{n}(\text { in })+b_{n}^{\dagger}(\text { in })\left(J_{n,-}-1\right) b_{n}(\text { in })\right]\right]:
\end{aligned}
$$

where the variables $J_{n, \zeta}$ are sources for the electron $(\zeta=+)$ or positron $(\zeta=-)$ in operators, $Z$ is a normalization factor (the partition function), and $: \cdots:$ here and in what follows means the normal form with respect to those creation and annihilation operators that are situated inside the colons.

Using the canonical transformation (79), found in the Appendix, we can express the inoperators in term of the out-operators and obtain $\check{R}(J)$ (and the corresponding $\check{\rho}$ ) in terms of the out operators, [15. Thus,

$\check{R}(J)=Z^{-1}(J)\left|c_{\mathrm{v}}\right|^{2} \operatorname{det}(1+\kappa A B)^{\kappa} \underline{\check{R}}(J)$,

$\underline{\check{R}}(J)=: \exp \left[-a^{\dagger}\right.$ (out) $\left(1-D_{+}\right) a($ out $)-b^{\dagger}$ (out) $\left(1-D_{-}\right) b$ (out) $-a^{\dagger}$ (out) $C^{\dagger} b^{\dagger}$ (out) $-b$ (out) $C a($ out $\left.)\right]:$,

$D_{+}=w(+\mid+)(1+\kappa A B)^{-1} \mathbb{J}_{+} w(+\mid+)^{\dagger}, \quad D_{-}^{T}=w(-\mid-)^{\dagger} \mathbb{J}_{-}(1+\kappa B A)^{-1} w(-\mid-), \quad A(J)=\mathbb{J}_{+} B^{\dagger} \mathbb{J}_{-}$,

$C=w(-\mid-)^{\dagger} \mathbb{J}_{-} B(1+\kappa A B)^{-1} \mathbb{J}_{+} w(+\mid+)^{\dagger}+\kappa w(+-\mid 0)^{\dagger}, \quad B=\kappa w(0 \mid-+), \mathbb{J}_{m n, \zeta}=\delta_{m n} J_{n, \zeta}$,

where $\kappa=+1$ for the Fermi case and $\kappa=-1$ for the Bose case. The normalization factor $Z$ has the form

$$
Z(J)=\exp \left\{\kappa \sum_{n, \zeta}\left[\ln \left(1+\kappa J_{n, \zeta}\right)\right]\right\}=\prod_{n, \zeta}\left[1+\kappa J_{n, \zeta}\right]^{\kappa} .
$$

In what follows, we work with two important cases of the general density operator that correspond to the initial vacuum state and to the initial thermal state.

(a) Setting $J=0$ and using the well-known formula [22], we obtain the density operator $\check{\rho}(0)$ that corresponds to the initial vacuum state

$$
\left.\check{\rho}(0)=: \exp \left\{-\sum_{n}\left[a_{n}^{\dagger} \text { (in) } a_{n} \text { (in) }+b_{n}^{\dagger}(\text { in }) b_{n}(\text { in })\right]\right\}:=\mid 0, \text { in }\right\rangle\langle 0, \text { in }| .
$$

From Eqs. (11) we obtain this operator in terms of the out-operators

$$
\begin{gathered}
\check{\rho}(0)=\left|c_{\mathrm{v}}\right|^{2}: \exp \left\{-\sum_{n}\left[a_{n}^{\dagger}(\text { out }) a_{n}(\text { out })+b_{n}^{\dagger}(\text { out }) b_{n}(\text { out })\right.\right. \\
\left.\left.+\kappa a_{n}^{\dagger} \text { (out) } w(+-\mid 0)_{n n} b_{n}^{\dagger} \text { (out) }+\kappa b_{n}(\text { out }) w(+-\mid 0)_{n n}^{\dagger} a_{n}(\text { out })\right]\right\}: .
\end{gathered}
$$

Differential mean numbers $N_{n, \zeta}(0 \mid$ in $)$ of in electrons and positrons in the state $\check{\rho}(0)$ are zero,

$$
N_{n,+}(0 \mid \text { in })=\operatorname{tr} \check{\rho}(0) a_{n}^{\dagger}(\text { in }) a_{n}(\text { in })=0, \quad N_{n,-}(0 \mid \text { in })=\operatorname{tr} \check{\rho}(0) b_{n}^{\dagger}(\text { in }) b_{n}(\text { in })=0,
$$

whereas differential mean numbers $N_{n, \zeta}(0 \mid$ out $)$ of out electrons and positrons in the state $\check{\rho}(0)$

$$
N_{n,+}(0 \mid \text { out })=\operatorname{tr} \check{\rho}(0) a_{n}^{\dagger} \text { (out) } a_{n} \text { (out), } \quad N_{n,-}(0 \mid \text { out })=\operatorname{tr} \check{\rho}(0) b_{n}^{\dagger} \text { (out) } b_{n} \text { (out), }
$$

are equal and have the form

$$
N_{n,+}(0 \mid \text { out })=N_{n,-}(0 \mid \text { out })=N_{n}(0 \mid \text { out }), N_{n}(0 \mid \text { out })=\frac{\left|w(+-\mid 0)_{n n}\right|^{2}}{1+\kappa\left|w(+-\mid 0)_{n n}\right|^{2}},
$$


(b) To obtain the density operator $\check{\rho}(\beta)$ that corresponds to the thermal initial state, one has to set $J_{n, \zeta}=J_{n, \zeta}(\beta)$,

$$
J_{n, \zeta}(\beta)=e^{-E_{n, \zeta}}, \quad E_{n, \zeta}=\beta\left(\varepsilon_{n, \zeta}-\mu_{\zeta}\right),
$$

where $\varepsilon_{n, \zeta}$ are energies of electrons $(\zeta=+)$ or positrons $(\zeta=-)$ with quantum numbers $n ; \mu_{\zeta}$ are the corresponding chemical potentials, and $\beta=\Theta^{-1}$, where $\Theta$ is the absolute temperature [15. It can be checked that an explicit expression for $\check{\rho}(\beta)$ in terms of the in operators is

$$
\begin{aligned}
& \check{\rho}(\beta)=Z_{\mathrm{gr}}^{-1} \exp \left[-\beta\left(\check{H}-\sum_{\zeta} \mu_{\zeta} \check{N}_{\zeta}\right)\right], \\
& Z_{\mathrm{gr}}=\exp \left[\kappa \sum_{n \zeta} \ln \left(1+\kappa e^{-E_{n, \zeta}}\right)\right] .
\end{aligned}
$$

The quantity $Z_{\mathrm{gr}}$ is the partition function of the grand canonical ensemble, $\check{H}$ is the Hamiltonian of the system (written in terms of in-operators),

$$
\check{H}=\sum_{n}\left[a_{n}^{\dagger}(\text { in }) \varepsilon_{n,+} a_{n}(\text { in })+b_{n}^{\dagger}(\text { in }) \varepsilon_{n,-} b_{n}(\text { in })\right],
$$

and

$$
\check{N}_{+}=\sum_{n}\left[a_{n}^{\dagger}(\text { in }) a_{n}(\text { in })\right], \quad \check{N}_{-}=\sum_{n}\left[b_{n}^{\dagger}(\text { in }) b_{n}(\text { in })\right]
$$

are operators of numbers of in-electrons and in-positrons, respectively.

Let $\check{\rho}$ be the general density matrix for an arbitrary initial state, $N_{n, \zeta}(\cdots \mid i n)$ be differential mean numbers of in electrons or positrons in the state $\check{\rho}$, and $N_{n, \zeta}(\cdots \mid$ out $)$ be differential mean numbers of out electrons or positrons in the state $\check{\rho}$,

$$
\begin{aligned}
& N_{n,+}(\cdots \mid \text { in })=\operatorname{tr} \check{\rho} a_{n}^{\dagger}(\text { in }) a_{n}(\text { in }), \quad N_{n,-}(\cdots \mid \text { in })=\operatorname{tr} \check{\rho} b_{n}^{\dagger}(\text { in }) b_{n}(\text { in }), \\
& \left.N_{n,+}(\cdots \mid \text { out })=\operatorname{tr} \check{\rho} a_{n}^{\dagger}(\text { out }) a_{n} \text { (out }\right), \quad N_{n,-}(\cdots \mid \text { out })=\operatorname{tr} \check{\rho} b_{n}^{\dagger}(\text { out }) b_{n}(\text { out }) .
\end{aligned}
$$

Calculating the traces in the in basis, one can see [15] that

$$
N_{n, \zeta}(\cdots \mid \text { out })=N_{n, \zeta}(\cdots \mid \text { in })+N_{n}(0 \mid \text { out })\left\{1-\kappa\left[N_{n,+}(\cdots \mid \text { in })+N_{n,-}(\cdots \mid \text { in })\right]\right\} .
$$

In particular, differential mean numbers $N_{n, \zeta}(\beta \mid i n)$ of in-electrons or positrons in the state $\check{\rho}(\beta)$ are the well-known Fermi-Dirac $(\kappa=+1)$ or Bose-Einstein $(\kappa=-1)$ distributions,

$$
\begin{aligned}
& N_{n,+}(\beta \mid \text { in })=\operatorname{tr} \check{\rho}(\beta) a_{n}^{\dagger}(\text { in }) a_{n}(\text { in })=\left(e^{E_{n,+}}+\kappa\right)^{-1}, \\
& N_{n,-}(\beta \mid \text { in })=\operatorname{tr} \check{\rho}(\beta) b_{n}^{\dagger}(\text { in }) b_{n}(\text { in })=\left(e^{E_{n,-}}+\kappa\right)^{-1} .
\end{aligned}
$$

Differential mean numbers $N_{n, \zeta}(\beta \mid$ out $)$ of out electrons or positrons in the state $\check{\rho}(\beta)$ follow immediately from (9).

\section{Reduced density operators for electron and positron subsystems}

At any fixed time instant, the complete system of quantum electrons and positrons can be conditionally divided into two subsystems: a system of electrons and a system of positrons. Let us suppose that the external electric field is switched off at some sufficiently long time 
instant $t_{2}$ in such a way that at $t_{\text {out }}>t_{2}$ no particle creation occurs and both subsystems are spatially separated. Thus, the particle creation effect by the time-dependent uniform electric field provides a real division of the complete quantum field system into the two subsystems. We can introduce the so-called reduced density operators $\check{\rho}_{ \pm}$of the electron subsystem and of the positron subsystem. These operators are defined as follows:

$\check{\rho}_{+}=\operatorname{tr}_{-} \check{\rho}=\sum_{M=0}^{\infty} \sum_{\{m\}}(M !)^{-1}{ }_{b}\langle 0$, out $| b_{m_{M}}($ out $) \cdots b_{m_{1}}($ out $)|\check{\rho}| b_{m_{1}}^{\dagger}$ (out) $\cdots b_{m_{M}}^{\dagger}($ out $) \mid 0$, out $\rangle_{b}$,
$\check{\rho}_{-}=\operatorname{tr}_{+} \check{\rho}=\sum_{M=0}^{\infty} \sum_{\{m\}}(M !)^{-1}{ }_{a}\langle 0$, out $| a_{m_{M}}($ out $) \cdots a_{m_{1}}$ (out) $|\check{\rho}| a_{m_{1}}^{\dagger}$ (out) $\cdots a_{m_{M}}^{\dagger}($ out $) \mid 0$, out $\rangle_{a}$,

where $\check{\rho}$ is the density operator of the complete system, $\mid 0$, out $\rangle_{a}$ and $\mid 0$, out $\rangle_{b}$ are electron and positron vacua, respectively, $\left(a_{m} \text { (out) } \mid 0 \text {, out }\right\rangle_{a}=0, b_{m}$ (out) $\mid 0$, out $\rangle_{b}=0$, $\mid 0$, out $\rangle=$ $\mid 0$, out $\rangle_{a} \otimes \mid 0$, out $\left.\rangle_{b}\right)$ and $\operatorname{tr}_{ \pm}$are the so-called reduced traces. Obviously, the reduced density operators $\check{\rho}_{ \pm}$describe mixed states.

The reduced density operators $\check{\rho}_{ \pm}$can be obtained from the reduced generating operators $\check{R}_{ \pm}(J)$ which are defined as:

$$
\check{R}_{ \pm}(J)=\operatorname{tr}_{\mp} \check{R}(J) .
$$

In terms of the out-operators these have the form

$$
\begin{aligned}
& \check{R}_{+}(J)=Z_{+}^{-1}(J): \exp \left\{-\sum_{n} a_{n}^{\dagger}(\text { out })\left[1-K_{+}(J)\right]_{n n} a_{n} \text { (out) }\right\}:, \\
& \check{R}_{-}(J)=Z_{-}^{-1}(J): \exp \left\{-\sum_{n} b_{n}^{\dagger}(\text { out })\left[1-K_{-}(J)\right]_{n n} b_{n} \text { (out) }\right\}:, \\
& K_{ \pm}(J)=D_{ \pm}+C^{\dagger}\left(1+\kappa D_{\mp}^{T}\right)^{-\kappa} C, \\
& Z_{ \pm}^{-1}(J)=Z^{-1}(J)\left|c_{\mathrm{v}}\right|^{2} \operatorname{det}(1+\kappa A B)^{\kappa} \operatorname{det}\left(1+\kappa D_{\mp}\right)^{\kappa} .
\end{aligned}
$$

The reduced generating operators $\check{R}_{ \pm}(J)$ allow one to obtain the reduced density operators $\check{\rho}_{ \pm}$for different initial states of the system. Consider as before two important cases.

(a) By setting $J=0$ in (13) we obtain the reduced density operators $\check{\rho}_{\zeta}(0)=\check{R}_{\zeta}(0)$ for both subsystems in the case when the complete system was in the vacuum state at the initial time instant. Taking into account that

$$
\begin{aligned}
& K_{ \pm}(0)=|w(+-\mid 0)|^{2}=P(+-\mid 0) P_{\mathrm{v}}^{-1}, \\
& \left.Z_{ \pm}^{-1}(0)=\left|c_{\mathrm{v}}\right|^{2}=P_{\mathrm{v}}, \quad P(+-\mid 0)=\mid\langle 0, \text { out }| a_{n} \text { (out) } b_{n} \text { (out) } \mid 0, \text { in }\right\rangle\left.\right|^{2},
\end{aligned}
$$

where $P(+-\mid 0)$ and $P_{\mathrm{v}}$ are probabilities of pair creation and the vacuum-to-vacuum transition, respectively, we obtain explicit expressions for $\check{\rho}_{\zeta}(0)$ :

$$
\begin{aligned}
& \check{\rho}_{+}(0)=\check{R}_{+}(0)=\left|c_{\mathrm{v}}\right|^{2}: \exp \left\{-\sum_{n} a_{n}^{\dagger}(\text { out })\left[1-P(+-\mid 0) P_{\mathrm{v}}^{-1}\right]_{n n} a_{n}(\text { out })\right\}:, \\
& \check{\rho}_{-}(0)=\check{R}_{-}(0)=\left|c_{\mathrm{v}}\right|^{2}: \exp \left\{-\sum_{n} b_{n}^{\dagger}(\text { out })\left[1-P(+-\mid 0) P_{\mathrm{v}}^{-1}\right]_{n n} b_{n}(\text { out })\right\}: .
\end{aligned}
$$

It should be noted that reduced density operators (14) were originally obtained in Ref. [21].

(b) By setting the sources $J$ in expression (13) equal to $J_{n, \zeta}(\beta)$ according to Eqs. (6), we see that the reduced generating operators (13) become the reduced density operators $\check{\rho}_{\zeta}(\beta)$ of the system that was in thermal equilibrium at the initial time instant $\check{R}_{+}(J)=\check{\rho}_{+}(\beta)$ and $\check{R}_{-}(J)=\check{\rho}_{-}(\beta)$. 


\section{Decoherence in course of the evolution}

\subsection{General}

In the previous sections we considered the case where the information loss was due to the averaging over one of the subsystems of electrons or positrons. However, information loss can also occur due to the interaction of the quantum system with classical (or semiclassical) objects, or, in other words, due to decoherence. One can imagine two possible scenarios for this: It can happen first during intermediate measurements by a classical tool and, second, as a result of collisions of particles with some semiclassical objects (for example, well-known impurities in the graphene). For us, there is no difference which of the mechanisms is implemented, so in what follows we talk about an intermediate measurement by a classical tool as a source of the decoherence.

Consider the case when the unitary evolution of the system is interrupted by a single intermediate measurement. The external field starts to act at the time instant $t_{\text {in }}$, the system is evolving in a unitary way from $t_{\text {in }}$ to $t_{1}$, during time $T_{1}$, then at $t_{1}$ a decoherence takes place, and then again the unitary evolution proceeds from $t_{1}$ to $t_{\text {out }}$ during time $T_{2}$. In this case, if we consider the Heisenberg picture, the out-set of creation and annihilation operators for electrons and positrons of the interval $T_{1}$ is the in set of the interval $T_{2}$.

Suppose that during time interval $T_{1}$ the system is described by density operator $\check{\rho}(0)$, i.e. the system is in the vacuum state at initial time instant $t_{\text {in }}$. Differential mean numbers of electrons and positrons at the time instant $t_{1}$ are the numbers of electrons or positrons created by the external field from the vacuum $N_{n}(0 \mid$ out $)$ (5). The electrons and positrons created in pairs by the external field are entangled.

During the time interval $T_{2}$ the system is described by the density operator which we denote by $\check{\rho}^{\prime}$. The latter in terms of the in set of creation-annihilation operators for electrons and positrons must describe the system without quantum correlations between the electrons and positrons created (i.e. new "initial" state of the system in the time interval $T_{2}$ is the state without any entanglement).

Such an operator can be obtained by using the von Neumann reduction principle 23 . Let a system be in a pure state that is described by a state vector $|\psi\rangle$, or equivalently by a density operator $\hat{\rho}$ that is in such a case the projector, $\hat{\rho}=\hat{P}_{\psi}=|\psi\rangle\langle\psi|$. In addition, let $\hat{R}$ be a self-adjoint observable of the system. In the simplest case, when this observable has a nondegenerate discrete spectrum the following spectral decomposition holds $\hat{R}=$ $\sum_{\alpha} r_{\alpha} P_{\varphi_{\alpha}}$, where $r_{\alpha}$ are possible eigenvalues of the observable, and $P_{\varphi_{\alpha}}$ are projectors on to the corresponding eigenvectors $\left|\varphi_{\alpha}\right\rangle, \hat{P}_{\varphi_{\alpha}}=\left|\varphi_{\alpha}\right\rangle\left\langle\varphi_{\alpha}\right|$. When measuring the observable $\hat{R}$, we obtain the eigenvalues $r_{\alpha}$ with the probabilities $\left|\left\langle\varphi_{\alpha} \mid \psi\right\rangle\right|^{2}=\left\langle\varphi_{\alpha}\left|\hat{P}_{\psi}\right| \varphi_{\alpha}\right\rangle=\left\langle\varphi_{\alpha}|\hat{\rho}| \varphi_{\alpha}\right\rangle$, and just after the measurement the state vector $|\psi\rangle$ is reduced to the vector $\left|\varphi_{\alpha}\right\rangle$, or the density operator $\hat{\rho}$ is reduced to the operator $\hat{\rho}^{\prime}=\hat{P}_{\varphi_{\alpha}}$. A more general case, where the system is in a mixed state, is described by the density operator $\hat{\rho}$ with a simple discrete spectrum, $\hat{\rho}=\sum_{n} \lambda_{n} P_{\psi_{n}}, P_{\psi_{n}}=\left|\psi_{n}\right\rangle\left\langle\psi_{n}\right|, \lambda_{n}$ being statistical weights of the corresponding states $P_{\psi_{n}}$, and $\hat{R}$ being the above-mentioned observable. Then the measurement is presented as follows. The eigenvalues $r_{\alpha}$ are measured with the probabilities

$$
\sum_{n} \lambda_{n}\left|\left\langle\varphi_{\alpha} \mid \psi_{n}\right\rangle\right|^{2}=\left\langle\varphi_{\alpha}|\hat{\rho}| \varphi_{\alpha}\right\rangle,
$$

and just after the measurement the density operator $\hat{\rho}$ is reduced to the operator $\hat{\rho}^{\prime}$,

$$
\hat{\rho}^{\prime}=\sum_{\alpha}\left\langle\varphi_{\alpha}|\hat{\rho}| \varphi_{\alpha}\right\rangle \hat{P}_{\varphi_{\alpha}} .
$$

The density operator $\check{\rho}(0)$ is

$$
\check{\rho}(0)=\mid 0, \text { in }\rangle\langle 0, \text { in }| .
$$


The in vacuum $\mid 0$, in $\rangle$ is connected to the out-vacuum $\mid 0$, out $\rangle$ by relation (80). Then density operator $\check{\rho}(0)$ can be presented as

$$
\check{\rho}(0)=V \mid 0, \text { out }\rangle\langle 0, \text { out }| V^{\dagger} .
$$

We are interested in the case of a uniform external field, which does not mix different quantum modes. Then amplitudes (78) are diagonal. Thus, it is possible to factorize $V$ defined by (80) as

$$
V=\prod_{n} V_{n}, \quad V_{n}=v_{4 n} v_{3 n} v_{2 n} v_{1 n}
$$

where

$v_{1 n}=\exp \left\{-\kappa b_{n}\right.$ (out) $w(0 \mid-+)_{n n} a_{n}$ (out) $\}, v_{2 n}=\exp \left\{a_{n}^{\dagger}\right.$ (out) $[\ln w(+\mid+)]_{n n} a_{n}$ (out) $\}$,

$v_{3 n}=\exp \left\{-\kappa b_{n}\right.$ (out) $[\ln w(-\mid-)]_{n n} b_{n}^{\dagger}$ (out) $\}, v_{4 n}=\exp \left\{-\kappa a_{n}^{\dagger}\right.$ (out) $w(+-\mid 0)_{n n} b_{n}^{\dagger}$ (out) $\}$.

Making use of the explicit form of $V_{n}$ and definition (80) we can write

$$
\left.\left.\mid 0, \text { in }\rangle=c_{\mathrm{v}} \prod_{n} \sum_{m=0} \frac{(-1)^{m}}{m !}\left[\kappa w(+-\mid 0)_{n n} a_{n}^{\dagger}(\text { out }) b_{n}^{\dagger} \text { (out }\right)\right]^{m} \mid 0, \text { out }\right\rangle,
$$

and then we can easily calculate $c_{\mathrm{v}}$,

$$
c_{\mathrm{v}}=\prod_{n}\left[w(-\mid-)_{n n}\right]^{-\kappa} .
$$

Thus, density operators $\check{\rho}(0)$ (16) can be represented as

$$
\begin{aligned}
& \check{\rho}(0)=\left|c_{\mathrm{v}}\right|^{2} \prod_{n}\left(\sum_{m=0}^{\infty} \frac{\left[-\kappa w(+-\mid 0)_{n n} a_{n}^{\dagger}(\text { out }) b_{n}^{\dagger}(\text { out })\right]^{m}}{m !}\right) \\
& \times \check{P}_{0} \prod_{n^{\prime}}\left(\sum_{m^{\prime}=0}^{\infty} \frac{\left[-\kappa w(+-\mid 0)_{n^{\prime} n^{\prime}}^{\dagger} b_{n^{\prime}}(\text { out }) a_{n^{\prime}} \text { (out) }\right]^{m^{\prime}}}{m^{\prime} !}\right),
\end{aligned}
$$

where $\check{P}_{0}=\mid 0$, out $\rangle\langle 0$, out $|$.

\subsection{Measurement of differential mean numbers in the system}

Suppose that we are going to measure the physical quantity, which is the number of particles, in the state $\check{\rho}(0)$ of the system under consideration. The operator corresponding to such physical quantity is $\check{N}$ (out),

$$
\check{N}(\text { out })=\sum_{n, \zeta} \check{N}_{n, \zeta}(\text { out })=\sum_{n}\left[a_{n}^{\dagger}(\text { out }) a_{n}(\text { out })+b_{n}^{\dagger}(\text { out }) b_{n}(\text { out })\right] .
$$

Its eigenstates are mutually orthonormal vectors of the form

$$
\begin{aligned}
& \left.\left.\mid s, \text { out }\rangle=\mid\{i, l\}_{L P}, \text { out }\right\rangle_{a} \otimes \mid\{j, k\}_{K Q}, \text { out }\right\rangle_{b}, \\
& \left.\left.\mid\{i, l\}_{L P}, \text { out }\right\rangle_{a}=\frac{\left[a_{i_{1}}^{\dagger}(\text { out })\right]^{l_{1}}}{\sqrt{l_{1} !}} \cdots \frac{\left[a_{i_{P}}^{\dagger} \text { (out) }\right]^{l_{P}}}{\sqrt{l_{P} !}} \mid 0, \text { out }\right\rangle_{a}, \\
& \left.\left.\mid\{j, k\}_{K Q}, \text { out }\right\rangle_{b}=\frac{\left[b_{j_{1}}^{\dagger} \text { (out) }\right]^{k_{1}}}{\sqrt{k_{1} !}} \cdots \frac{\left[b_{j_{Q}}^{\dagger} \text { (out) }\right]^{k_{Q}}}{\sqrt{k_{Q} !}} \mid 0, \text { out }\right\rangle_{b}, \\
& L=0,1,2, \ldots, \quad P=1,2, \ldots L, \quad i=i_{1}, \ldots, i_{P}, \quad l_{1}+l_{2}+\ldots+l_{P}=L, \\
& K=0,1,2, \ldots, \quad Q=1,2, \ldots K, \quad j=j_{1}, \ldots, j_{Q}, \quad k_{1}+k_{2}+\ldots+k_{Q}=K,
\end{aligned}
$$


such that its eigenvalues are

$$
\hat{N}(\text { out }) \mid s, \text { out }\rangle=(L+K) \mid s, \text { out }\rangle,
$$

where $s$ is the full set of quantum numbers $K, L,\{i\},\{j\}, P$, and $Q$ and $\mid\{i, l\}_{L P}$, out $\rangle_{a}$ is a state with $L$ electrons distributed in $P$ groups $i_{1}, \ldots, i_{P}$, with $l_{1}$ electrons in the group $i_{1}, l_{2}$ electrons in the group $i_{2}$, and so on. Analogously, $\mid\{j, k\}_{K Q}$, out $\rangle_{b}$ is a state with $K$ positrons distributed in $Q$ groups $j_{1}, \ldots, j_{Q}$, with $k_{1}$ positrons in group $j_{1}, k_{2}$ positrons in group $j_{2}$, and so on.

According to von Neumann 23, the density operator $\check{\rho}(0)$ is after such measurement reduced to the operator $\check{\rho}_{N}$ of the form

$$
\left.\check{\rho}_{N}=\sum_{s}\langle s, \text { out }|\check{\rho}(0)| s, \text { out }\rangle \check{P}_{s}, \quad \check{P}_{s}=\mid s, \text { out }\right\rangle\langle s, \text { out }| .
$$

Due to the structure of the density operator $\check{\rho}(0)$ given by Eq. (20), the weights $\langle s$, out $|\check{\rho}(0)| s$, out $\rangle$ are non-zero only when the states $\mid s$, out $\rangle$ are states with an integer number of pairs. Thus, we obtain

$$
\begin{aligned}
& \left.\check{\rho}_{N}=\left|c_{\mathrm{v}}\right|^{2} \sum_{f} W_{f} \check{P}_{f}, \quad \sum_{f}=\sum_{M=0}^{\infty} \sum_{Z=1}^{M} \sum_{\{m, n\}}, \quad \check{P}_{f}=\mid f, \text { out }\right\rangle\langle f, \text { out }|, \\
& W_{f}=\left|w(+-\mid 0)_{n_{1} n_{1}}\right|^{2 m_{1}} \ldots\left|w(+-\mid 0)_{n_{Z} n_{Z}}\right|^{2 m_{Z}}, \quad m_{1}+m_{2}+\ldots+m_{z}=M, \\
& \left.\mid f, \text { out }\rangle=\frac{\left.\left[a_{n_{1}}^{\dagger} \text { out }\right) b_{n_{1}}^{\dagger} \text { (out) }\right]^{m_{1}}}{m_{1} !} \cdots \frac{\left.\left[a_{n_{Z}}^{\dagger} \text { (out }\right) b_{n_{Z}}^{\dagger}(\text { out })\right]^{m_{Z}}}{m_{Z} !} \mid 0, \text { out }\right\rangle,
\end{aligned}
$$

where $f$ is a complete set of quantum numbers $M, Z,\{m\}$, and $\{n\}$ and $\mid f$, out $\rangle$ is a state with the total number of pairs $M$ distributed in $Z$ groups, $m_{1}$ pairs being in the group $n_{1}, m_{2}$ pairs being in the group $n_{2}$ and so on. Unlike (20), the latter expression contains only terms diagonal in $f$. Thus, the measurement destroy nondiagonal terms of the density operator (20).

Let us now calculate the reduced (in the sense of Sec. 3) operators $\left[\check{\rho}_{N}\right]_{\zeta}$ :

$$
\begin{aligned}
{\left[\check{\rho}_{N}\right]_{\zeta} } & =\operatorname{tr}_{-\zeta} \check{\rho}_{N}=\left|c_{\mathrm{v}}\right|^{2} \sum_{f} W_{f} \operatorname{tr}_{-\zeta} \check{P}_{f}, \\
\operatorname{tr}_{-} \check{P}_{f} & \left.=\frac{\left[a_{n_{1}}^{\dagger}(\text { out })\right]^{m_{1}}}{\sqrt{m_{1} !}} \cdots \frac{\left[a_{n_{Z}}^{\dagger}(\text { out })\right]^{m_{Z}}}{\sqrt{m_{Z} !}} \mid 0, \text { out }\right\rangle_{a a}\langle 0, \text { out }| \frac{\left.\left[a_{n_{Z}} \text { (out }\right)\right]^{m_{Z}}}{\sqrt{m_{Z} !}} \cdots \frac{\left[a_{n_{1}} \text { (out) }\right]^{m_{1}}}{\sqrt{m_{1} !}} \\
\operatorname{tr}_{+} P_{f} & \left.=\frac{\left[b_{n_{1}}^{\dagger} \text { (out) }\right]^{m_{1}}}{\sqrt{m_{1} !}} \cdots \frac{\left[b_{n_{Z}}^{\dagger}(\text { out })\right]^{m_{Z}}}{\sqrt{m_{Z} !}} \mid 0, \text { out }\right\rangle_{b b}\langle 0, \text { out }| \frac{\left[b_{n_{Z}}(\text { out })\right]^{m_{Z}}}{\sqrt{m_{Z} !}} \cdots \frac{\left[b_{n_{1}}(\text { out })\right]^{m_{1}}}{\sqrt{m_{1} !}}
\end{aligned}
$$

On the other hand, one can calculate the reduced density operators $\check{\rho}_{\zeta}(0)$ by taking reduced traces (11) of the operator (20) to verify that they have exactly the same form,

$$
\left[\check{\rho}_{N}\right]_{\zeta}=\check{\rho}_{\zeta}(0) \text {. }
$$

\subsection{Measurements of differential mean numbers in the subsystems}

Suppose now that we measure the number of either electrons or positrons. The corresponding operators of these physical quantities are

$$
\begin{aligned}
& \check{N}_{+}(\text {out })=\sum_{n} \check{N}_{n,+}(\text { out })=\sum_{n} a_{n}^{\dagger}(\text { out }) a_{n}(\text { out }), \\
& \check{N}_{-}(\text {out })=\sum_{n} \check{N}_{n,-}(\text { out })=\sum_{n} b_{n}^{\dagger}(\text { out }) b_{n}(\text { out })
\end{aligned}
$$


The spectra of the operators (25) are

$$
\begin{aligned}
& \left.\left.\left.\mid s_{+}, \text {out }\right\rangle_{a}=\mid\{i, l\}_{L P}, \text { out }\right\rangle_{a}=\frac{\left[a_{i_{1}}^{\dagger}(\text { out })\right]^{l_{1}}}{\sqrt{l_{1} !}} \cdots \frac{\left[a_{i_{P}}^{\dagger} \text { (out) }\right]^{l_{P}}}{\sqrt{l_{P} !}} \mid 0, \text { out }\right\rangle_{a}, \\
& L=0,1,2, \ldots, \quad P=1,2, \ldots L, \quad i=i_{1}, \ldots, i_{P}, \quad l_{1}+l_{2}+\ldots+l_{P}=L, \\
& \left.\left.\left.\mid s_{-}, \text {out }\right\rangle_{b}=\mid\{j, k\}_{K Q}, \text { out }\right\rangle_{b}=\frac{\left[b_{j_{1}}^{\dagger} \text { (out) }\right]^{k_{1}}}{\sqrt{k_{1} !}} \cdots \frac{\left[b_{j_{Q}}^{\dagger}(\text { out })\right]^{k_{Q}}}{\sqrt{k_{Q} !}} \mid 0, \text { out }\right\rangle_{b}, \\
& K=0,1,2, \ldots, \quad Q=1,2, \ldots K, \quad j=j_{1}, \ldots, j_{Q}, \quad k_{1}+k_{2}+\ldots+k_{Q}=K, \\
& \left.\left.\left.\left.\left.\hat{N}_{+}(\text {out }) \mid s_{+}, \text {out }\right\rangle_{a}=L \mid s_{+}, \text {out }\right\rangle_{a}, \quad \hat{N}_{-} \text {(out }\right) \mid s_{-}, \text {out }\right\rangle_{b}=K \mid s_{-}, \text {out }\right\rangle_{b} .
\end{aligned}
$$

The states $\mid\{i, l\}_{L P}$, out $\rangle_{a}$ and $\mid\{j, k\}_{K Q}$, out $\rangle_{b}$ are defined in the same way as in the previous section.

The density operators after such measurements, which we denote by $\check{\rho}_{N_{+}}$and $\check{\rho}_{N_{-}}$, respectively, have the form

$$
\begin{aligned}
& \left.\check{\rho}_{N_{+}}=\sum_{s+}{ }_{a}\left\langle s_{+}, \text {out }|\check{\rho}(0)| s_{+}, \text {out }\right\rangle_{a} P_{s+}, \quad P_{s+}=\mid s_{+}, \text {out }\right\rangle_{a a}\left\langle s_{+}, \text {out }\right|, \quad \sum_{s+}=\sum_{L=0}^{\infty} \sum_{P=1}^{L} \sum_{\{i, l\}}, \\
& \left.\check{\rho}_{N_{-}}=\sum_{s-}{ }_{b}\left\langle s_{-}, \text {out }|\check{\rho}(0)| s_{-}, \text {out }\right\rangle_{b} P_{s-}, \quad P_{s_{-}}=\mid s_{-}, \text {out }\right\rangle_{b b}\left\langle s_{-}, \text {out }\right|, \quad \sum_{s-}=\sum_{K=0}^{\infty} \sum_{Q=1}^{K} \sum_{\{j, k\}} .
\end{aligned}
$$

Let us now calculate the quantities ${ }_{a}\left\langle s_{+} \text {, out }|\check{\rho}(0)| s_{+}, \text {out }\right\rangle_{a} P_{s+}$ and ${ }_{b}\left\langle s_{-} \text {, out }|\check{\rho}(0)| s_{-}, \text {out }\right\rangle_{b} P_{s-}$. Due to the structure of $\check{\rho}(0)$ they are equal and have the form

$$
\begin{aligned}
& { }_{a}\left\langle s_{+}, \text {out }|\check{\rho}(0)| s_{+}, \text {out }\right\rangle_{a} P_{s_{+}}=_{b}\left\langle s_{-}, \text {out }|\check{\rho}(0)| s_{-}, \text {out }\right\rangle_{b} P_{s_{-}} \\
& \left.=\left|c_{\mathrm{V}}\right|^{2} \frac{\left.\left.\left[-\kappa w(+-\mid 0)_{i_{1} i_{1}} a_{i_{1}}^{\dagger} \text { (out }\right) b_{i_{1}}^{\dagger} \text { (out }\right)\right]^{l_{1}}}{l_{1} !} \cdots \frac{\left.\left[-\kappa w(+-\mid 0)_{i_{p} i_{p}} a_{i_{p}}^{\dagger} \text { (out }\right) b_{i_{p}}^{\dagger}(\text { out })\right]^{l_{p}}}{l_{p} !} \mid 0, \text { out }\right\rangle \\
& \times\langle 0, \text { out }| \frac{\left[-\kappa w(+-\mid 0)_{i_{p} i_{p}}^{\dagger} b_{i_{p}}(\text { out }) a_{i_{p}}(\text { out })\right]^{l_{p}}}{l_{p} !} \cdots \frac{\left.\left.\left[-\kappa w(+-\mid 0)_{i_{1} i_{1}}^{\dagger} b_{i_{1}} \text { (out }\right) a_{i_{1}} \text { (out }\right)\right]^{l_{1}}}{l_{1} !} .
\end{aligned}
$$

It is not difficult to see that density operators $\check{\rho}_{N_{+}}$and $\check{\rho}_{N-}$ have exactly the same form as in (23), namely, they are sums over all possible projectors on states with an integer number of pairs

$$
\check{\rho}_{N+}=\check{\rho}_{N-}=\check{\rho}_{N} .
$$

Thus, we stress that measurements of $N, N_{+}$and $N_{-}$produce the same reductions. The reduced density operators $\left[\check{\rho}_{N+}\right]_{\zeta}=\operatorname{tr}_{-\zeta} \check{\rho}_{N+}$ and $\left[\check{\rho}_{N-}\right]_{\zeta}=\operatorname{tr}_{-\zeta} \check{\rho}_{N+}$ are equal to the reduced density operators $\check{\rho}_{\zeta}(0)$ given in (14).

It is also an interesting task to consider the case when the unitary evolution of the system is interrupted by multiple measurements. However, because there are significant technical difficulties, this problem is not considered in this paper.

\section{Entropy and entanglement of electron and positron subsystems}

As already said in the Introduction, the measure of the information loss in a quantum state $\check{\rho}$ can be identified with the entropy of such a state, namely, with the von Neumann 
information entropy $S[23$,

$$
S(\check{\rho})=-k_{B} \operatorname{tr} \check{\rho} \ln \check{\rho} .
$$

Let $\hat{\rho}\left(t_{\text {in }}\right)=\check{\rho}(\beta)$, where $\check{\rho}(\beta)$ is given by (7), then

$$
S(\check{\rho}(\beta))=k_{B}\left[\ln Z_{g r}+\sum_{n \zeta} E_{n, \zeta} N_{n, \zeta}(\beta \mid \text { in })\right],
$$

The corresponding differential mean numbers $N_{n, \zeta}(\beta \mid i n)$ are Fermi-Dirac or Bose-Einstein distributions, given by (10). The entropy (30) can be written in terms of the Bose (Fermi) occupation number alone, if we take into account that

$$
e^{-E_{n, \zeta}}=\frac{N_{n, \zeta}(\beta \mid \text { in })}{1-\kappa N_{n, \zeta}(\beta \mid \text { in })} .
$$

Then

$$
S(\check{\rho}(\beta))=-k_{B} \sum_{n \zeta}\left\{\kappa\left[1-\kappa N_{n, \zeta}(\beta \mid \text { in })\right] \ln \left[1-\kappa N_{n, \zeta}(\beta \mid \text { in })\right]+N_{n, \zeta}(\beta \mid \text { in }) \ln N_{n, \zeta}(\beta \mid \text { in })\right\} .
$$

This expression has a form similar to expressions for entropy of the grand canonical ensemble for Fermi- and Bose-particles [24].

Especially interesting information is obtained by calculating the von Neumann information entropy of the reduced density operators of both the electron and positron subsystems $S\left(\hat{\rho}_{ \pm}\right)$,

$$
S\left(\hat{\rho}_{ \pm}\right)=-k_{B} \operatorname{tr}_{ \pm}\left(\hat{\rho}_{ \pm} \ln \hat{\rho}_{ \pm}\right) .
$$

According to the general theory they coincide $S\left(\hat{\rho}_{+}\right)=S\left(\hat{\rho}_{-}\right)$and can be treated as a measure of the quantum entanglement of these subsystems.

It is also known that one can recognize entanglement by evaluating the so-called Schmidt measure, which is the trace of the squared reduced density operators [25]

$$
\tilde{S}\left(\hat{\rho}_{ \pm}\right)=-\operatorname{tr}\left[\left(\hat{\rho}_{ \pm}\right)^{2}\right] .
$$

Let us calculate the entropy for both the electron and positron subsystems in two important cases of the vacuum initial state and the thermal initial state that are described by the reduced density operators $\check{\rho}_{\zeta}(0)$ and $\check{\rho}_{\zeta}(\beta)$.

\subsection{Vacuum initial state}

The entropy for the reduced density operator of the system with an initial vacuum state has the form

$$
S\left(\check{\rho}_{\zeta}(0)\right)=-k_{B} \operatorname{tr}_{\zeta}\left(\check{\rho}_{\zeta}(0) \ln \check{\rho}_{\zeta}(0)\right) .
$$

The term $\ln \check{\rho}_{\zeta}(0)$ on the right-hand side of (35) can be written as

$$
\begin{aligned}
& \ln \check{\rho}_{+}(0)=\ln \left[\left|c_{\mathrm{v}}\right|^{2}: \exp \left\{-\sum_{n} a_{n}^{\dagger} \text { (out) }\left(1-P(+-\mid 0) P_{\mathrm{v}}^{-1}\right)_{n n} a_{n} \text { (out) }\right\}:\right], \\
& \ln \check{\rho}_{-}(0)=\ln \left[\left|c_{\mathrm{v}}\right|^{2}: \exp \left\{-\sum_{n} b_{n}^{\dagger}(\text { out })\left(1-P(+-\mid 0) P_{\mathrm{v}}^{-1}\right)_{n n} b_{n}(\text { out })\right\}:\right] .
\end{aligned}
$$


Transforming the normal-form exponents into ordinary exponents (see, for example, [15]) and recalling that $\left|c_{\mathrm{v}}\right|^{2}=P_{\mathrm{v}}$, we obtain

$$
\begin{aligned}
& \ln \check{\rho}_{+}(0)=\ln P_{\mathrm{v}}+\sum_{n} a_{n}^{\dagger}(\text { out }) \ln \left[P(+-\mid 0) P_{\mathrm{v}}^{-1}\right]_{n n} a_{n}(\text { out }), \\
& \ln \check{\rho}_{-}(0)=\ln P_{\mathrm{v}}+\sum_{n} b_{n}^{\dagger}(\text { out }) \ln \left[P(+-\mid 0) P_{\mathrm{v}}^{-1}\right]_{n n} b_{n} \text { (out). }
\end{aligned}
$$

Taking into account that the matrices $P(+-\mid 0) P_{\mathrm{v}}^{-1}$ are diagonal, one can rewrite (35) as

$$
\begin{gathered}
S\left(\check{\rho}_{+}(0)\right)=-k_{B}\left\{\ln P_{\mathrm{v}}+\sum_{n} \operatorname{tr}_{+}\left(\check{\rho}_{+}(0) a_{n}^{\dagger}(\text { out }) a_{n}(\text { out })\right) \ln \left[P(+-\mid 0) P_{\mathrm{v}}^{-1}\right]_{n n}\right\}, \\
S\left(\check{\rho}_{-}(0)\right)=-k_{B}\left\{\ln P_{\mathrm{v}}+\sum_{n} \operatorname{tr}_{-}\left(\check{\rho}_{-}(0) b_{n}^{\dagger}(\text { out }) b_{n}(\text { out })\right) \ln \left[P(+-\mid 0) P_{\mathrm{v}}^{-1}\right]_{n n}\right\},
\end{gathered}
$$

where $\operatorname{tr}_{+} \check{\rho}_{+}(0) a_{n}^{\dagger}$ (out) $a_{n}$ (out) $=N_{n}(0 \mid$ out $)$ and $\operatorname{tr}_{-} \check{\rho}_{-}(0) b_{n}^{\dagger}($ out $) b_{n}$ (out) $=N_{n}(0 \mid$ out $)$ are differential mean numbers of out-electrons and out-positrons, respectively. They obviously coincide. Thus, the entropy takes the form

$$
S\left(\check{\rho}_{\zeta}(0)\right)=-k_{B}\left\{\ln P_{\mathrm{v}}+\sum_{n} N_{n}(0 \mid \text { out })\left[\ln P(+-\mid 0) P_{\mathrm{v}}^{-1}\right]_{n n}\right\} .
$$

One can use the pair creation probability and the vacuum-to-vacuum probability written in terms of differential mean numbers (see, for example, [14])

$$
P(-+\mid 0)_{n, n^{\prime}}=\delta_{n, n^{\prime}} \frac{P_{\mathrm{v}} N_{n}(0 \mid \text { out })}{1-\kappa N_{n}(0 \mid \text { out })}, \quad P_{\mathrm{v}}=\exp \left\{\kappa \sum_{n} \ln \left[1-\kappa N_{n}(0 \mid \text { out })\right]\right\},
$$

to obtain

$$
\begin{aligned}
& S\left(\check{\rho}_{\zeta}(0)\right)=\sum_{n} S\left(\check{\rho}_{n, \zeta}(0)\right), \\
& S\left(\check{\rho}_{n, \zeta}(0)\right)=-k_{B}\left\{\kappa\left[1-\kappa N_{n}(0 \mid \text { out })\right] \ln \left[1-\kappa N_{n}(0 \mid \text { out })\right]+N_{n}(0 \mid \text { out }) \ln N_{n}(0 \mid \text { out })\right\} .
\end{aligned}
$$

Let us consider the Schmidt entanglement measure (34),

$$
\tilde{S}\left(\check{\rho}_{\zeta}(0)\right)=-\operatorname{tr}\left[\check{\rho}_{\zeta}(0)\right]^{2} .
$$

Here

$$
\begin{aligned}
& {\left[\check{\rho}_{+}(0)\right]^{2}=P_{\mathrm{v}}^{2}\left\{: \exp \left[-\sum_{n} a_{n}^{\dagger}(\text { out })\left(1-P(+-\mid 0) P_{\mathrm{v}}^{-1}\right)_{n n} a_{n}(\text { out })\right]:\right\}^{2},} \\
& {\left[\check{\rho}_{-}(0)\right]^{2}=P_{\mathrm{v}}^{2}\left\{: \exp \left[-\sum_{n} b_{n}^{\dagger}(\text { out })\left(1-P(+-\mid 0) P_{\mathrm{v}}^{-1}\right)_{n n} b_{n}(\text { out })\right]:\right\}^{2} .}
\end{aligned}
$$

Using the relation (here $D$ and $\tilde{D}$ are matrices)

$: \exp \left[-a^{\dagger}\right.$ (out) $D a($ out $\left.)\right]:: \exp \left[-a^{\dagger}\right.$ (out) $\tilde{D} a($ out $\left.)\right]:=: \exp \left[-a^{\dagger}\right.$ (out) $(D+\tilde{D}-D \tilde{D}) a($ out $\left.)\right]:$, 
we obtain

$$
\begin{aligned}
& {\left[\check{\rho}_{+}(0)\right]^{2}=P_{\mathrm{v}}^{2}: \exp \left\{\sum_{n} a_{n}^{\dagger} \text { (out) }\left[\left(P(+-\mid 0) P_{\mathrm{v}}^{-1}\right)^{2}-1\right]_{n n} a_{n} \text { (out) }\right\}:,} \\
& {\left[\check{\rho}_{-}(0)\right]^{2}=P_{\mathrm{v}}^{2}: \exp \left\{\sum_{n} b_{n}^{\dagger} \text { (out) }\left[\left(P(+-\mid 0) P_{\mathrm{v}}^{-1}\right)^{2}-1\right]_{n n} b_{n}(\text { out })\right\}: .}
\end{aligned}
$$

Calculating the traces in (42) taking into account (40), we finally obtain

$$
\begin{aligned}
& \tilde{S}\left(\check{\rho}_{\zeta}(0)\right)=-P_{\mathrm{v}}^{2} \operatorname{det}\left[1+\kappa\left(P(+-\mid 0) P_{\mathrm{v}}^{-1}\right)^{2}\right]^{\kappa} \\
& =-\prod_{n}\left\{1-2 \kappa N_{n}(0 \mid \text { out })+(1+\kappa)\left[N_{n}(0 \mid \text { out })\right]^{2}\right\}^{\kappa} .
\end{aligned}
$$

\subsection{Thermal initial state}

The entropy for the operators $\check{\rho}_{\zeta}(\beta)$, which describe the system that has been in thermal equilibrium at the initial time instant, has the form

$$
S\left(\check{\rho}_{\beta, \zeta}\right)=-k_{B} \operatorname{tr}_{\zeta} \check{\rho}_{\zeta}(\beta) \ln \check{\rho}_{\zeta}(\beta) .
$$

Transforming the expressions $\ln \check{\rho}_{\zeta}(\beta)$ as

$$
\begin{aligned}
& \ln \check{\rho}_{+}(\beta)=\ln Z_{\zeta}\left(J_{\beta}\right)+\sum_{n} a_{n}^{\dagger}(\text { out }) \ln \left[K_{+}\left(J_{\beta}\right)\right]_{n n} a_{n}(\text { out }), \\
& \ln \check{\rho}_{-}(\beta)=\ln Z_{\zeta}\left(J_{\beta}\right)+\sum_{n} b_{n}^{\dagger} \text { (out) } \ln \left[K_{-}\left(J_{\beta}\right)\right]_{n n} b_{n} \text { (out), }
\end{aligned}
$$

one can write

$$
S\left(\check{\rho}_{\beta, \zeta}\right)=k_{B}\left\{\ln Z_{\zeta}\left(J_{\beta}\right)-\sum_{n} N_{n, \zeta}(\beta \mid \text { out })\left[\ln K_{\zeta}\left(J_{\beta}\right)\right]_{n n}\right\},
$$

where $N_{n, \zeta}(\beta \mid$ out $)$ are given by (9) with $N_{n, \zeta}(\cdots \mid$ in $)=N_{n, \zeta}(\beta \mid$ in $)$. One can express diagonal elements of $K_{\zeta}\left(J_{\beta}\right)$ in terms of the corresponding occupation numbers $N_{n, \zeta}(\beta \mid$ out),

$$
\left[K_{\zeta}\left(J_{\beta}\right)\right]_{n n}=\frac{N_{n, \zeta}(\beta \mid \text { out })}{1-\kappa N_{n, \zeta}(\beta \mid \text { out })}
$$

and do the same to $Z_{\zeta}\left(J_{\beta}\right)$ by means of the normalization condition $\left(\operatorname{tr}_{\zeta} \check{\rho}_{\beta, \zeta}=1\right)$

$$
Z_{\zeta}\left(J_{\beta}\right)=\exp \left\{-\kappa \sum_{n} \ln \left[1-\kappa N_{n, \zeta}(\beta \mid \text { out })\right]\right\},
$$

to rewrite the expression (49) for the entropy in the form

$$
\begin{aligned}
& S\left(\check{\rho}_{\zeta}(\beta)\right)=\sum_{n} S\left(\check{\rho}_{n, \zeta}(\beta)\right), \quad S\left(\check{\rho}_{n, \zeta}(\beta)\right) \\
& =-k_{B}\left\{\kappa\left[1-\kappa N_{n, \zeta}(\beta \mid \text { out })\right] \ln \left[1-\kappa N_{n, \zeta}(\beta \mid \text { out })\right]+N_{n, \zeta}(\beta \mid \text { out }) \ln N_{n, \zeta}(\beta \mid \text { out })\right\} .
\end{aligned}
$$

Considering expressions (52), (41), and (32) one can see that they all have similar forms.

Next, let us find the Schmidt measure for subsystems of positrons and electrons for the system with a thermal state as the initial time instant; subsystems of such a state are 
described by the reduced density operator $\check{\rho}_{\zeta}(\beta)$. The entanglement measure of the electron and positron subsystem is given by

$$
\tilde{S}\left(\check{\rho}_{\zeta}(\beta)\right)=-\operatorname{tr}\left[\check{\rho}_{\zeta}(\beta)\right]^{2},
$$

where the squares of the operators $\check{\rho}_{\zeta}(\beta)$ are

$$
\begin{aligned}
& {\left[\check{\rho}_{+}(\beta)\right]^{2}=Z_{+}^{-2}\left(J_{\beta}\right): \exp \left[\sum_{n} a_{n}^{\dagger}(\text { out })\left[K_{+}^{2}\left(J_{\beta}\right)-1\right]_{n n} a_{n}(\text { out })\right]:,} \\
& {\left[\check{\rho}_{-}(\beta)\right]^{2}=Z_{-}^{-2}\left(J_{\beta}\right): \exp \left[\sum_{n} b_{n}^{\dagger} \text { (out) }\left[K_{-}^{2}\left(J_{\beta}\right)-1\right]_{n n} b_{n}(\text { out })\right]:,}
\end{aligned}
$$

such that

$$
\tilde{S}\left(\check{\rho}_{\zeta}(\beta)\right)=-\prod_{n}\left\{1-2 \kappa N_{n, \zeta}(\beta \mid \text { out })+(1+\kappa)\left[N_{n, \zeta}(\beta \mid \text { out })\right]^{2}\right\}^{\kappa}
$$

\subsection{Entropy of measurement-reduced density operators}

The entropy of a density operator $\check{\rho}_{N}(22)$ has the form

$$
S\left(\check{\rho}_{N}\right)=-k_{B} \operatorname{tr} \check{\rho}_{N} \ln \check{\rho}_{N} .
$$

The representation (3) allows one to factorize the complete vacuum into the product of single-mode vacua

$$
\begin{aligned}
& \mid 0, \text { out }\rangle\left\langle 0, \text { out }\left|=\prod_{n}\right| 0, \text { out }\right\rangle_{n n}\langle 0, \text { out }|, \\
& \left.\left.a_{n} \text { (out) } \mid 0, \text { out }\right\rangle_{n}=0, \quad b_{n} \text { (out) } \mid 0, \text { out }\right\rangle_{n}=0 .
\end{aligned}
$$

Using this fact and the representation for $\left|c_{\mathrm{V}}\right|^{2}$ from (19), one can rewrite the density operator (22) as a product of single-mode density operators:

$$
\begin{aligned}
& \left.\check{\rho}_{N}=\prod_{n} \check{\rho}_{N, n}, \quad \operatorname{tr} \check{\rho}_{N, n}=1, \check{\rho}_{N, n}=\left|c_{\mathrm{v}}\right|_{n}^{2} \sum_{f=0} W_{f, n} \mid f, \text { out }\right\rangle_{n n}\langle f, \text { out }|, \\
& \left.\left.\left|c_{\mathrm{v}}\right|_{n}^{2}=\left|w(-\mid-)_{n n}\right|^{-2 \kappa}, \quad W_{f, n}=\left|w(+-\mid 0)_{n n}\right|^{2 f}, \quad \mid f, \text { out }\right\rangle_{n}=\frac{\left.\left[a_{n}^{\dagger}(\text { out }) b_{n}^{\dagger} \text { (out }\right)\right]^{f}}{f !} \mid 0, \text { out }\right\rangle_{n} .
\end{aligned}
$$

The quantities $\left|c_{\mathrm{v}}\right|_{n}^{2}$ and $\left|w(+-\mid 0)_{n n}\right|^{2}$ can be expressed via differential numbers $N_{n}(0 \mid$ out $)$ as

$$
\left|c_{\mathrm{v}}\right|_{n}^{2}=\left(1-\kappa N_{n}(0 \mid \text { out })\right)^{\kappa}, \quad\left|w(+-\mid 0)_{n n}\right|^{2}=\frac{N_{n}(0 \mid \text { out })}{1-\kappa N_{n}(0 \mid \text { out })} .
$$

Due to expression (58), the entropy (56) can be written as

$$
S\left(\check{\rho}_{N}\right)=-k_{B} \sum_{n} \operatorname{tr} \check{\rho}_{N, n} \ln \check{\rho}_{N, n} .
$$

To calculate the trace of the operator $\check{\rho}_{N, n} \ln \check{\rho}_{N, n}$, one can use the formal decomposition

$$
\check{\rho}_{N, n} \ln \check{\rho}_{N, n}=\check{\rho}_{N, n} \sum_{k=1}^{\infty} k^{-1}\left(\check{\rho}_{N, n}-1\right)^{k}=\sum_{k=1}^{\infty} k^{-1} \sum_{l=0}^{k} C_{k}^{l}\left(\check{\rho}_{N, n}\right)^{l+1}(-1)^{k-l}
$$


where $C_{k}^{l}$ are binomial coefficients. Due to the orthonormality of the states $\mid f$, out $\rangle_{n}$ the density operators $\left(\check{\rho}_{N, n}\right)^{l+1}$ have the form

$$
\left.\left(\check{\rho}_{N, n}\right)^{l+1}=\left|c_{\mathrm{v}}\right|_{n}^{2(l+1)} \sum_{f=0}^{\infty}\left(W_{f, n}\right)^{l+1} \mid f, \text { out }\right\rangle_{n n}\langle f, \text { out }| .
$$

Substituting (62) into (61), we obtain

$$
\left.\check{\rho}_{N, n} \ln \check{\rho}_{N, n}=\left|c_{\mathrm{V}}\right|_{n}^{2} \sum_{f=0}^{\infty} W_{f, n} \ln \left(\left|c_{\mathrm{V}}\right|_{n}^{2} W_{f, n}\right) \mid f, \text { out }\right\rangle_{n n}\langle f, \text { out }| .
$$

Then

$$
\operatorname{tr} \check{\rho}_{N, n} \ln \check{\rho}_{N, n}=N_{n}(0 \mid \text { out }) \ln N_{n}(0 \mid \text { out })+\kappa\left[1-\kappa N_{n}(0 \mid \text { out })\right] \ln \left[1-\kappa N_{n}(0 \mid \text { out })\right] .
$$

Thus, the entropy of the density operator (22) reads

$$
S\left(\check{\rho}_{N}\right)=-k_{B} \sum_{n}\left\{\kappa\left[1-\kappa N_{n}(0 \mid \text { out })\right] \ln \left[1-\kappa N_{n}(0 \mid \text { out })\right]+N_{n}(0 \mid \text { out }) \ln N_{n}(0 \mid \text { out })\right\} .
$$

The result has the same form as the entropy $S\left(\check{\rho}_{\zeta}(0)\right)$ given by (41). Thus, we can say that the measurement of $N, N_{+}$or $N_{-}$leads to the same information loss as a reduction over electrons or positrons.

It was shown in the Sec. 4 that reduction of the density operator $\check{\rho}_{N}$ over electrons and positrons transforms it in $\left[\check{\rho}_{N}\right]_{\zeta}=\check{\rho}_{\zeta}(0)$. This means that if one calculates the entropy of the density operator $\left[\check{\rho}_{N}\right]_{\zeta}$, one obtains the same expression (65) again. The conditional entropy [3] $S_{\text {cond }}=S\left(\check{\rho}_{N}\right)-S\left(\left[\check{\rho}_{N}\right]_{\zeta}\right)$, which is used as a measure of correlations between subsystems, is zero. This fact means that all quantum correlations between the electrons and positrons are lost due to decoherence, and there is no entanglement left after the measurement.

\section{T-constant external electric field}

To illustrate some of the above general formulas we consider the so-called $T$-constant electric field as an external background. Such a field acts only during a finite time $T$ and it is constant within this time interval. Using this field allows one to avoid troubles with the definition of in- and out-states inherent to external fields non-switched at $t \rightarrow \pm \infty$. Another important point is that this field produces a finite work in a finite space volume. Let us consider $d=(D+1)$-dimensional space; then the $T$-constant electric field $\mathbf{E}$ is acting during the time interval $T=t_{\text {out }}-t_{\text {in }}$,

$$
\mathbf{E}=(0, E(t), 0, \ldots, 0), E(t)=\left\{\begin{array}{l}
0, \quad-\infty<t \leq t_{\text {in }} \\
E>0, \quad t_{\text {in }}<t<t_{\text {out }}
\end{array},\right.
$$

Processes of pair creation in such a field were studied in Refs. [27, 28, 14, 26]). Similar to these works, we consider sufficiently large $T$.

Since there is no particle production after the time instant $t_{\text {out }}$, differential mean numbers of particles $N_{n, \zeta}(\cdots \mid$ out $)$ created in a given state $n=\mathbf{p}, r$ ( $\mathbf{p}$ is a $D$-dimensional vector of momentum and $r$ is spin) depend only on the time interval. The electric field acting during the sufficiently long time $T$ creates a considerable number of pairs only in a finite region in the momentum space. Since we suppose $T \gg \max \left\{1, E_{c} / E\right\}$, we need to consider only the range

$$
\left|p_{\perp}\right| \leq \sqrt{e E}[\sqrt{e E} T]^{1 / 2}, \quad-T / 2 \leq p_{1} / e E \leq T / 2
$$

in the momentum space, see 14 for details. Note that for the case $d=2$ there are no transversal components of momentum. 


\subsection{Vacuum initial state}

First let us consider the case when the system initially was in the vacuum state. For this case the differential mean numbers in the momentum range (67) are

$$
N_{n}(0 \mid \text { out })=e^{-\pi \lambda}, \quad \lambda=\left(p_{\perp}^{2}+m^{2}\right) / e E .
$$

They have the same form as in the case of the constant uniform electric field [21, 11] and are the same for bosons and fermions. The entropy (41) is expressed in terms of $N_{n}$ (0|out) and does not depend on the spin quantum number $r$, thus, the summation over the latter results in the factor $\gamma_{(d)}=2^{\left[\frac{d}{2}\right]-1}$.

First we consider the Dirac case with $\kappa=+1$ :

$$
S\left(\check{\rho}_{n, \zeta}(0)\right)=-k_{B}\left\{\left[1-N_{n}(0 \mid \text { out })\right] \ln \left[1-N_{n}(0 \mid \text { out })\right]+N_{n}(0 \mid \text { out }) \ln N_{n}(0 \mid \text { out })\right\} .
$$

For the case of electric field the mean number of particles created $N_{n}(0 \mid$ out $)$ can vary only within the range $(0,1)$ and depends only on thestrength of the external field. Expression (69) is symmetric with respect to $N_{n, \zeta}$ (0|out). It reaches maximum at $N_{n}(0 \mid$ out $)=1 / 2$ and turns to zero at $N_{n}(0 \mid$ out $)=1$ and $N_{n}(0 \mid$ out $)=0$. This fact can be interpreted as follows. In the case of $N_{n}$ (0|out) $=0$ there are no particles created by the external field and theinitial vacuum state in the mode remains unchanged. The case $N_{n}(0 \mid$ out $)=1$ corresponds to the situation when a particle is created with certainty. The maximum of (69), corresponding to $N_{n}(0 \mid$ out $)=1 / 2$, is associated with the state with the maximum amount of uncertainty.

Representing the logarithm in the first term of expression (69) as the Taylor series in powers of $N_{n}$ (0|out), we see that $S\left(\check{\rho}_{n, \zeta}(0)\right)$ is proportional to $N_{n}$ (0|out). The latter plays the role of the cut-off parameter for the integral over $p_{1}[14$. Thus, the summation over the quantum numbers can be reduced to an integration over momenta that satisfy restrictions (67),

$$
\sum_{n} \rightarrow \frac{\gamma_{(d)} V}{(2 \pi)^{d-1}} \int d \mathbf{p}
$$

where $V$ is the $D$-dimensional spatial volume. The mean numbers (68) do not depend on the longitudinal component of momentum. Outside of the range (67), the contribution to the integral is very small, and this allows us to extend the integration limits of $p_{\perp}$ to infinity. Integration over $p_{\perp}$ can be performed using the Taylor series. The result of the integration is

$$
S\left(\check{\rho}_{\zeta}(0)\right)=\gamma_{(d)} k_{B} \frac{(e E)^{\frac{d}{2}} T V}{(2 \pi)^{d-1}} A_{\text {Dirac }}\left(d, E_{c} / E\right),
$$

where the factor $T V$ can be considered as the $d$-dimensional volume. To get finite and correct expressions, one should use the volume normalization. The factor $A_{\text {Dirac }}\left(d, E_{c} / E\right)$ has the form

$$
\begin{aligned}
& A_{\text {Dirac }}\left(d, E_{c} / E\right)=\sum_{l=1}^{\infty} l^{-d / 2} \exp \left[-\pi l E_{c} / E\right] \\
& -\sum_{l=1}^{\infty} l^{-1}(l+1)^{(2-d) / 2} \exp \left[-\pi(l+1) E_{c} / E\right]+\left(\pi \frac{E_{c}}{E}+\frac{d-2}{2}\right) \exp \left(-\pi E_{c} / E\right) .
\end{aligned}
$$

It is possible to estimate the entropy in strong-field $E_{c} / E \ll 1$, critical-field $E_{c} / E=1$, and weak-feald $E_{c} / E \gg 1$ limits. For example, for a strong field with $d=4$ we have $A_{\text {Dirac }}(4,0)=\pi^{2} / 6$, for the critical field, we have $A_{\text {Dirac }}(4,1) \approx 0,22$. In the case of a weak field the entropy has a small value of the order of $\left(\pi E_{c} / E\right) \exp \left[-\pi E_{c} / E\right]$ for any $d$. For $d=3$ the following estimations hold $A_{\text {Dirac }}(3,0) \approx 0,93, A_{\text {Dirac }}(3,1) \approx 0,2$; for $d=2$ the factor $A(2,0)$ is a value of order of 1 , and $A(2,1)=e^{-\pi}$. 
Let us consider the KG case $(\kappa=-1)$,

$$
S\left(\check{\rho}_{n, \zeta}(0)\right)=k_{B}\left\{\left[1+N_{n}(0 \mid \text { out })\right] \ln \left[1+N_{n}(0 \mid \text { out })\right]-N_{n}(0 \mid \text { out }) \ln N_{n}(0 \mid \text { out })\right\} .
$$

Expression (71) just increases with $N_{n}$ (0|out). After summation over the quantum numbers, the entropy (39) takes the form

$$
S\left(\check{\rho}_{\zeta}(0)\right)=k_{B} \frac{(e E) d^{d / 2} T V}{(2 \pi)^{d-1}} A_{\mathrm{KG}}\left(d, E_{c} / E\right),
$$

where

$$
\begin{aligned}
& A_{\mathrm{KG}}\left(d, E_{c} / E\right)=\sum_{l=1}^{\infty} l^{-d / 2}(-1)^{l-1} \exp \left[-\pi l E_{c} / E\right] \\
& +\sum_{l=1}^{\infty} l^{-1}(l+1)^{(2-d) / 2}(-1)^{l-1} \exp \left[-\pi(l+1) E_{c} / E\right]+\left(\pi \frac{E_{c}}{E}+\frac{d-2}{2}\right) \exp \left(-\pi E_{c} / E\right) .
\end{aligned}
$$

The following are estimations for different field strengths: $A_{\mathrm{KG}}(4,0) \approx 2,21, A_{\mathrm{KG}}(4,1) \approx$ 0,$22 ; A_{\mathrm{KG}}(3,0) \approx 1,78, A_{\mathrm{KG}}(3,1) \approx 0,2 ; A_{\mathrm{KG}}(2,0) \approx 1, A_{\mathrm{KG}}(2,1) \approx e^{-\pi}$. In the case of weak field the entropy is a small value of the order of $\left(\pi E_{c} / E\right) \exp \left[-\pi E_{c} / E\right]$ for any $d$ again.

We have mentioned before that the entropy of the density operator $\check{\rho}_{N}$, given by (65), is exactly of the same form as the entropy of $\check{\rho}_{\zeta}(0)$, given by (41), hence all the considerations for the case with the intermediate measurement are the same.

\subsection{Mixed initial state}

We note that the entropy (52) of the system that has been in thermal equilibrium at the initial time instant is expressed in terms of differential mean numbers $N_{n, \zeta}(\beta \mid$ out) (9) of particles created by the external field, whereas initial differential numbers of particles are

$$
N_{n, \zeta}(\beta \mid \text { in })=\left[\exp \beta\left(\varepsilon_{n}-\mu\right)+\kappa\right]^{-1}, \quad \varepsilon_{n}=\sqrt{m^{2}+p_{\perp}^{2}+\left(p_{1}+e E T / 2\right)^{2}} .
$$

Let us discuss two cases, the first one being the case of low temperature

$$
\beta\left(\epsilon_{\perp}-\mu\right) \gg 1, \epsilon_{\perp}=\sqrt{m^{2}+p_{\perp}^{2}},
$$

when all the energies of the particles created with a given $p_{\perp}$ are considerably higher than the temperature, and the second being the case of high temperature $\beta e E T \ll 1$, when all the energies of the created particles are much lower than the temperature. We assume for simplicity that $e E T \gg \mu$ and that $T$ is sufficiently large to provide $(e E T)^{2} \gg m^{2}+p_{\perp}^{2}$.

In the low-temperature case, the number of particles created does not depend on the longitudinal momenta:

$$
N_{n, \zeta}(\beta \mid \text { in }) \approx \exp \left(-\beta \varepsilon_{n}\right) \rightarrow 0, \quad N_{n, \zeta}(\beta \mid \text { out }) \rightarrow N_{n, \zeta}(0 \mid \text { out }) .
$$

In this limit entropy $S\left(\check{\rho}_{n, \zeta}(\beta)\right)$ tends to that of the zero-temperature case (initial vacuum state). Then integration over transversal momenta can be done exactly as in the initial vacuum case.

Formal calculations of $N_{n, \zeta}$ ( $\beta \mid$ out) and of the entropy in the case of high temperature, $\beta e E T \ll 1$, are also quite simple. However, it was shown in Ref. 26 that in the Dirac case under such a condition the current density is much greater than the current density of particles created from the vacuum, due to the work of the external field performed over 
the particles [which was denoted by $\operatorname{Re}\left\langle j_{\mu}(t)\right\rangle_{\theta}^{c}$ in [26] already existing in the initial state. Therefore, in such a case the particle creation effect may be disregarded.

We note that the general form of the reduced density operators $\check{\rho}_{ \pm}$, given by Eq. (13), allows one to study the change of the entropy and the corresponding entanglement during many consecutive measurements. In this case the density operator $\check{\rho}_{N}(23)$ has to be considered as the initial state for the second stage of the evolution and so on. In the general case it is not simple to describe such a decoherence procedure for an arbitrary stage. However, as was mentioned above, if the mean numbers $N_{n}(0 \mid$ out $)$ are not small within a sufficiently large range of momenta, already on the second stage the particle-creation effect may be disregarded and the subsequent decoherence is described in the usual terms.

\section{Summary}

Using a general nonperturbative expression for the density operators (of quantized Dirac or KG fields), we derived their specific forms corresponding to different initial conditions. Applying a reduction procedure to specific density operators, we constructed mixed states of both electron and positron subsystems. Calculating the entropy of such states, we obtained the loss of information due to the reduction and, at the same time, the entanglement of electron and positron subsystems. We paid attention to the fact that any measurement in the system under consideration implies a decoherence and the corresponding modifications of the complete and the reduced density operators. We studied the results of such a decoherence and we related to it the loss of information by calculating the information entropy. To illustrate some of the obtained general results, we considered the slowly varying $T$-constant electric field as an external background. We derived the following conclusions. The entropy of any subsystem (of electrons or positrons) with the vacuum as the initial state is proportional to the factor $(e E)^{d / 2}$ and to the number of spin degrees of freedom $\gamma_{(d)}$. It grows linearly with the time of the field action $T$. The above behavior remains in the thermal case at low temperatures; in fact, here the entropy does not depend on the temperature.

Acknowledgements

SPG thanks FAPESP for support and University of São Paulo for hospitality. DMG is grateful to the Brazilian foundations FAPESP and CNPq for support. The work of SPG and DMG was also partially supported by the Tomsk State University Competitiveness Improvement Program. AAS thanks CAPES for support. The reported study of SPG, DMG, and AAS was partially supported by RFBR Project No. 15-02-00293a.

\section{A QED with strong electric-like background}

In this appendix, we consider briefly a special case of QFT with an unstable vacuum of the quantized Dirac or KG field with time-dependent electriclike background that is switched on and off at $t \rightarrow \pm \infty$. Quantization of this theory in terms of in- and out-electrons and positrons was elaborated in Refs. [13. Some results of this quantization necessary for us here are presented below.

We denote operators in the Schrödinger representation by a caret, e.g., $\hat{A}$, while operators in the Heisenberg representation are denoted by an inverted caret, g.e., $\check{A}$. In the Schrödinger picture one can define the following: at the initial time instant $t_{\text {in }}$, a set of creation and annihilation operators $a_{n}^{\dagger}\left(t_{\text {in }}\right)$ and $a_{n}\left(t_{\text {in }}\right)$ of electrons and similar operators $b_{n}^{\dagger}\left(t_{\text {in }}\right)$ and $b_{n}\left(t_{\text {in }}\right)$ of positrons such that the corresponding vacuum at $t_{\text {in }}$ is $\left|0, t_{\text {in }}\right\rangle$; at the final time instant $t_{\text {out }}$, a set of creation and annihilation operators $a_{n}^{\dagger}\left(t_{\text {out }}\right), a_{n}\left(t_{\text {out }}\right)$, of electrons and similar operators $b_{n}^{\dagger}\left(t_{\text {out }}\right), b_{n}\left(t_{\text {out }}\right)$ of positrons, such that the corresponding vacuum at $t_{\text {out }}$ is $\left|0, t_{\text {out }}\right\rangle$,

$$
a_{n}\left(t_{\text {in }}\right)\left|0, t_{\text {in }}\right\rangle=b_{n}\left(t_{\text {in }}\right)\left|0, t_{\text {in }}\right\rangle=0, a_{n}\left(t_{\text {out }}\right)\left|0, t_{\text {out }}\right\rangle=b_{n}\left(t_{\text {out }}\right)\left|0, t_{\text {out }}\right\rangle=0 \quad \forall n .
$$


The probability amplitude for the transition from an initial state to a final state $M_{\text {in } \rightarrow \text { out }}$ has the following form in the Schrödinger picture:

$$
M_{\text {in } \rightarrow \text { out }}=\left\langle t_{\text {out }}\left|U\left(t_{\text {out }}, t_{\text {in }}\right)\right| t_{\text {in }}\right\rangle,
$$

where $U\left(t, t^{\prime}\right)$ is a unitary evolution operator of the system. The density operator of an initial state $\hat{\rho}\left(t_{\text {in }}\right)$ is given as an operator-valued function of the creation and annihilation operators of electrons (positrons) at the initial time instant

$$
\hat{\rho}\left(t_{\text {in }}\right)=\rho_{\text {in }}\left(a^{\dagger}\left(t_{\text {in }}\right), a\left(t_{\text {in }}\right), b^{\dagger}\left(t_{\text {in }}\right), b\left(t_{\text {in }}\right)\right) .
$$

The mean value of a physical quantity $F$ at the final time instant reads

$$
\left\langle F\left(t_{\text {out }}\right)\right\rangle=\operatorname{tr} \hat{\rho}\left(t_{\text {out }}\right) \hat{F}\left(t_{\text {out }}\right),
$$

where $\hat{\rho}(t)$ is the density operator in the Schrödinger representation at time instant $t$, and the designation tr stands for the complete trace,

$$
\hat{\rho}\left(t_{\text {out }}\right)=U\left(t_{\text {out }}, t_{\text {in }}\right) \hat{\rho}\left(t_{\text {in }}\right) U^{\dagger}\left(t_{\text {out }}, t_{\text {in }}\right) .
$$

In order to pass to the Heisenberg picture we define the finite-time evolution operators $\Omega_{( \pm)}$,

$$
\begin{aligned}
& \Omega_{(+)}=U\left(0, t_{\text {in }}\right), \Omega_{(-)}=U\left(0, t_{\text {out }}\right), U\left(t_{\text {out }}, t_{\text {in }}\right)=\Omega_{(-)}^{\dagger} \Omega_{(+)}, \\
& \check{\rho}=\hat{\rho}(0)=\Omega_{(+)} \hat{\rho}\left(t_{\text {in }}\right) \Omega_{(+)}^{\dagger}=\Omega_{(-)} \hat{\rho}\left(t_{\text {out }}\right) \Omega_{(-)}^{\dagger} ;
\end{aligned}
$$

a set of creation and annihilation operators $a_{n}^{\dagger}$ (in) and $a_{n}$ (in) of in-electrons, similar operators $b_{n}^{\dagger}$ (in) and $b_{n}$ (in) of in positrons, and the corresponding in vacuum $\mid 0$, in $\rangle$; and a set of creation and annihilation operators $a_{n}^{\dagger}$ (out), $a_{n}$ (out), of out electrons, similar operators $b_{n}^{\dagger}$ (out) and $b_{n}$ (out) of out positrons, and the corresponding out vacuum $\mid 0$, out $\rangle$,

$$
\begin{aligned}
& \left.\{a(\text { in }), \ldots\}=\Omega_{(+)}\left\{a\left(t_{\text {in }}\right), \cdots\right\} \Omega_{(+)}^{\dagger}, \mid 0, \text { in }\right\rangle=\Omega_{(+)}\left|0, t_{\text {in }}\right\rangle, \\
& \left.\{a(\text { out }), \ldots\}=\Omega_{(-)}\left\{a\left(t_{\text {out }}\right), \ldots\right\} \Omega_{(-)}^{\dagger}, \mid 0, \text { out }\right\rangle=\Omega_{(-)}\left|0, t_{\text {out }}\right\rangle, \\
& \left.M_{\text {in } \rightarrow \text { out }}=\left\langle 0, t_{\text {out }}\left|\cdots a\left(t_{\text {in }}\right) \Omega_{(-)}^{\dagger} \Omega_{(+)} a_{n}^{\dagger}\left(t_{\text {in }}\right) \cdots\right| 0, t_{\text {in }}\right\rangle=\langle 0, \text { out }| \cdots a(\text { out }) a_{n}^{\dagger}(\text { in }) \cdots \mid 0, \text { in }\right\rangle, \\
& \left.c_{v}=\left\langle 0, t_{\text {out }}\left|U\left(t_{\text {out }}, t_{\text {in }}\right)\right| 0, t_{\text {in }}\right\rangle=\langle 0, \text { out }| 0, \text { in }\right\rangle .
\end{aligned}
$$

All the information concerning the processes of particle creation, annihilation and scattering is contained in the elementary probability amplitudes

$$
\begin{aligned}
& \left.w(+\mid+)_{m n}=c_{v}^{-1}\langle 0, \text { out }| a_{m} \text { (out) } a_{n}^{\dagger}(\text { in }) \mid 0, \text { in }\right\rangle, \\
& \left.w(-\mid-)_{n m}=c_{v}^{-1}\langle 0, \text { out }| b_{m} \text { (out) } b_{n}^{\dagger} \text { (in) } \mid 0, \text { in }\right\rangle, \\
& \left.w(0 \mid-+)_{n m}=c_{v}^{-1}\langle 0, \text { out }| b_{n}^{\dagger} \text { (in) } a_{m}^{\dagger} \text { (in) } \mid 0, \text { in }\right\rangle, \\
& \left.w(+-\mid 0)_{m n}=c_{v}^{-1}\langle 0, \text { out }| a_{m} \text { (out) } b_{n} \text { (out) } \mid 0, \text { in }\right\rangle .
\end{aligned}
$$

The amplitudes (78) can be calculated with the help of certain appropriate sets of solutions of the corresponding relativistic wave equation with an external field (Klein-Gordon, Dirac, and so on) (see [13]). We are interested in the case of a uniform external field, which does not mix different quantum modes. Thus, in this paper the amplitudes (78) are diagonal in quantum numbers,

$w(\zeta \mid \zeta)_{m n}=\delta_{m n} w(\zeta \mid \zeta)_{n n}, w(0 \mid-+)_{n m}=\delta_{m n} w(0 \mid-+)_{n n}, w(+-\mid 0)_{n m}=\delta_{m n} w(+-\mid 0)_{n n}$. 
The sets of in- and out-operators are related to each other by a linear canonical transformation 22, which can be written in terms of the amplitudes (78) 2

$$
\begin{aligned}
& a(\text { out })=\left[w(+\mid+)^{\dagger}\right]^{-1} a(\text { in })-\kappa w(+-\mid 0)[w(-\mid-)]^{-1} b^{\dagger}(\text { in }), \\
& b^{\dagger}(\text { out })=\left[w(+\mid+)^{\dagger}\right]^{-1} w(+-\mid 0)^{\dagger} a(\text { in })+[w(-\mid-)]^{-1} b^{\dagger}(\text { in }),
\end{aligned}
$$

and by its Hermitian conjugate. As it has been demonstrated [13]), such a relation is given by a unitary operator $V$,

$$
\left.\left.V\{a(\text { out }), \ldots\} V^{\dagger}=\{a(\text { in }), \ldots\}, \quad \mid 0, \text { in }\right\rangle=V \mid 0, \text { out }\right\rangle,
$$

which has the form $V=v_{4} v_{3} v_{2} v_{1}$,

$$
\begin{aligned}
& v_{1}=\exp \{-\kappa b(\text { out }) w(0 \mid-+) a(\text { out })\}, v_{2}=\exp \left\{a^{\dagger}(\text { out }) \ln w(+\mid+) a(\text { out })\right\}, \\
& v_{3}=\exp \left\{-\kappa b(\text { out }) \ln w(-\mid-) b^{\dagger}(\text { out })\right\}, v_{4}=\exp \left\{-\kappa a^{\dagger} \text { (out) } w(+-\mid 0) b^{\dagger} \text { (out) }\right\} .
\end{aligned}
$$

Using this expression for $V$, one can find

$$
c_{v}=\langle 0, \text { out }|V| 0, \text { out }\rangle=\exp \{-\kappa \operatorname{tr} \ln w(-\mid-)\} .
$$

\section{References}

[1] J. S. Bell, Speakable and Unspeakable in Quantum Mechanics (Cambridge University Press, New York, 1987).

[2] J. Preskill, Lecture notes on quantum computation, available at http://www.theory.caltech.edu/people/preskill/ph219/\#lecture (1997-1999).

[3] M. A. Nielsen and I.L. Chuang, Quantum Computation and Quantum Information (Cambridge University Press, Cambridge, 2000).

[4] R. Alicki and M. Fannes, Quantum Dynamical Systems (Oxford University Press, New York, 2001).

[5] S-Y Lin, C-H Chou, B.L. Hu, Phys. Rev. D 81, 084018 (2010).

[6] Z. Ebadi and B. Mirza, Ann. Phys. (N.Y.) 351, 363 (2014).

[7] C. Kiefer, Phys. Rev. D 46, 1658 (1992).

[8] S. Habib, Y. Kluger, E. Mottola, and J. P. Paz, Phys. Rev. Lett. 76, 4660 (1996).

[9] S. W. Hawking, Commun. Math. Phys. 43, 199 (1975).

[10] I. D. Novikov and V. P. Frolov, Fund. Theor. Phys. 27, 190 (1989).

[11] A. I. Nikishov, Zh. Eksp. Teor. Fiz. 57, 1210 (1969) [Sov. Phys. JETP 30, 660 (1970)]; Quantum Electrodynamics of Phenomena in Intense Fields, Proceedings of the P.N. Lebedev Physical Instute Vol. 111 (Nauka, Moscow, 1979), p. 153.

\footnotetext{
${ }^{2}$ We use condensed notation, for example, $b w(0 \mid-+) a=\sum_{n, m} b_{n} w(0 \mid-+)_{n m} a_{m}$.
} 
[12] N.D. Birrell and P.C.W. Davies, Quantum Fields in Curved Space (Cambridge University Press, Cambridge, 1982); A.A. Grib, S.G. Mamaev, and V.M. Mostepanenko, Vacuum Quantum Effects in Strong Fields (Friedmann Laboratory, St. Petersburg, 1994); R. Ruffini, G. Vereshchagin and S. Xue, Phys. Rep. 487, 1 (2010).

[13] D.M. Gitman, J. Phys. A 10, 2007 (1977); E.S. Fradkin and D.M. Gitman, Fortschr. Phys. 29, 381 (1981); E.S. Fradkin, D.M. Gitman and S.M. Shvartsman, Quantum Electrodynamics with Unstable Vacuum (Springer, Berlin, 1991).

[14] S.P. Gavrilov and D.M. Gitman, Phys. Rev. D 53, 7162 (1996).

[15] S.P. Gavrilov, D.M. Gitman, and J.L. Tomazelli, Nucl. Phys. B 795, 645 (2008).

[16] J. Schwinger, Phys. Rev. 82, 664 (1951).

[17] G. V. Dunne, Eur. Phys. J. D 55, 327 (2009).

[18] N. Vandecasteele, A. Barreiro, M. Lazzeri, A. Bachtold and F. Mauri, Phys. Rev. B 82, 045416 (2010).

[19] S. P. Gavrilov, D. M. Gitman, and N. Yokomizo, Phys. Rev. D 86, 125022 (2012).

[20] M.I. Katsnelson, G.E. Volovik, and M.A. Zubkov, Ann. Phys. (N.Y.) 336, 36 (2013).

[21] D.M. Gitman and V.P. Frolov, Sov. J. Nucl. Phys. 28, 552 (1978); J. Phys. A 15, 1329 (1978).

[22] F. A. Berezin, The Method of Second Quantization (Nauka, Moscow, 1965) [(Academic Press, New York, 1966)].

[23] J. von Neumann, Mathematische Grundlagen der Quantenmechanik (Verlag von Julius Springer, Berlin, 1932).

[24] L. D. Landau and E. M. Lifshitz, Statistical Physics (Addison-Wesley, Reading, MA, 1969).

[25] J. Eisert, H.J. Briegel, Phys. Rev. A 64, 022306 (2001).

[26] S. P. Gavrilov and D. M. Gitman, Phys. Rev. D 78, 045017 (2008).

[27] V.G. Bagrov, D.M. Gitman, and Sh.M. Shvartsman, Zh. Eksp. Teor. Fiz. 68, 392 (1975) [Sov. Phys. JETP 41, 191 (1975)].

[28] D.M. Gitman, V.M. Shachmatov, and Sh. M. Shvartsman, Izv. Vyssh. Uchebn. Zaved. Fiz. (Sov. Phys. Journ.) 18, No. 4, 23 (1975). 